\title{
Mobile ECG Monitoring for Atrial Fibrillation Management: A Literature Review
}

\author{
Vincent Pearson ${ }^{1,2}$ \\ ${ }^{1}$ College of Nursing and Health Sciences, Flinders University, South Australia \\ ${ }^{2}$ Department of Heart and Lung, Central Adelaide Local Health Network \\ Corresponding author: Vincent Pearson (e-mail: vincent.pearson@ @linders.edu.au).
}

\begin{abstract}
Atrial fibrillation is the most prevalent arrhythmia associated with the risk of developing stroke and heart failure. Catheter ablation and electrical cardioversion are standard interventional procedures to treat atrial fibrillation. Patients are recommended to undertake regular heart rhythm assessment after the procedure due to the high recurrence rate of atrial fibrillation. Multiple mobile applications with high sensitivity and accuracy rates in rhythm analysis have been developed to support on-demand rhythm monitoring. This literature review aims to provide an overview of emerging evidence about the impact of mobile ECG monitoring on AF management. Keywords and index terms used in the search strategy include "atrial fibrillation" AND "mhealth" OR "mobile health" OR "mobile app" OR "mobile application" OR smartphone OR mobile AND "electrocardiography", "electrocardiogram" OR "ECG" OR "monitoring". A search using all identified keywords and index terms was undertaken across the following databases: PubMed, CINAHL, and EMBASE. Incorporating the novel mobile ECG monitoring technology in the guideline-adherent integrated AF care model may reduce rehospitalisation, improve HRQoL, and better patient clinical outcomes. Mobile applications with ECG monitoring functions can provide user-friendly on-demand rhythm monitoring, facilitating the early detection of recurrent AF post-ablation.
\end{abstract}

INDEX TERMS Atrial Fibrillation; Electrocardiography; Mobile Application; Smartphone; Telemedicine.

\section{INTRODUCTION}

Atrial Fibrillation (AF) is the most prevalent arrhythmia [1]. The global burden of AF has doubled since 1990 [2]. It is predicted that the global burden of AF will increase by at least $60 \%$ in 2050 [3]. The prevalence of AF increases with advancing age. According to the Australian Institute of Health and Welfare [4], AF prevalence among Australians over 55 is estimated to be around 5\%. Besides, AF is associated with the risk of developing stroke and heart failure [5]. In Australia, the annual health care expense on diagnosing and treating $\mathrm{AF}$ is estimated to be $\$ 881$ million [4].

Atrial fibrillation is often treated with antiarrhythmic medications or interventional procedures. Standard interventional procedures that aim to restore sinus rhythm include catheter ablation and electrical cardioversion [6]. However, the recurrence rate is high (41\% to 54\%) after the procedure [7]. Professional guidelines recommend routine rhythm monitoring and symptom assessment to guide $\mathrm{AF}$ management [6]. Traditionally, patients have 12-lead electrocardiogram (ECG) assessments at an outpatient clinic or general practice at regular intervals [8]. Due to the intermittent nature of $\mathrm{AF}$, it can be challenging to detect this arrhythmia during each short office visit [9].

The 12-lead ECG is essential for diagnosing and detecting AF [10]. However, collecting electric conduction data from all 12 leads is not always necessary for informing $\mathrm{AF}$ treatment decisions [10]. Ambulatory ECG monitoring devices such as Holter monitors utilising a small number of classic ECG leads can be ordered for eligible patients for up to 30 days [6]. However, the discomfort from wearing the device and relatively high healthcare costs are two common barriers to implementing this monitoring method [9].

There has been a significant advancement in information technology in the past decade [11]. According to the Australian Bureau of Statistics [12], 86\% of Australian 
households have internet access, and 91\% of them use smartphones to access the internet. Furthermore, there has also been increasing use of mobile health for chronic disease management [11]. World Health Organization [13] defines mobile health as a therapeutic practice supported by mobile devices such as smartphones and mobile accessories. Because of the reliance on ECGs for arrhythmia detection and management, there has been a push to develop mobile applications to provide on-demand rhythm monitoring [14]. Due to being user-friendly and cost-effective, mobile applications with ECG monitoring functions are likely suitable for on-demand rhythm monitoring for $\mathrm{AF}$ management [15]. Multiple mobile applications with high sensitivity and accuracy rates in AF rhythm analysis have been developed and tested [15]. There are two ways to obtain a single-lead ECG using a mobile application. First, Photo Plethysmography (PPG) technology can detect the heart rhythm by analysing skin colour and light absorption from the tissue when the user places his or her finger over the camera lens for up to 60 seconds [16]. The variation of light intensity via transmission through or reflection from the tissue provides information about the heart rhythm's rate, strength, and regularity [16]. Second, a portable handheld smartphone ECG recorder can obtain a single-lead ECG [1]. Mobile health applications with mathematical algorithms can analyse the single-lead ECG and provide feedback to the mobile user [16]. Some mobile applications can even transmit the user's rhythm information to a medical centre for the ECG to be reviewed by a specialist [17].

Despite the potential clinical benefits, there is a paucity of studies that formally evaluate the impact of mobile health applications with ECG monitoring for AF management. A systematic review conducted by Lane, et al. [18] evaluated the impact of mobile health applications on AF management. However, the systematic review excluded mobile health applications that support ECG monitoring of AF. This literature review aims to provide an overview of emerging evidence about the impact of mobile ECG monitoring on AF management. It is essential to understand the scope, clinical benefits and acceptability of the novel mobile ECG monitoring technology before incorporating it into the $\mathrm{AF}$ management plan. Thus, this review will analyse and synthesise the evidence about the utilisation of mobile ECG monitoring for AF management. It will also explore the key concepts that underpin the research field of mobile ECG monitoring for AF management and identify knowledge gaps in mobile ECG monitoring for AF management and areas for future research.

\section{METHOD}

The search strategy aimed to locate published studies regarding the impact of mobile ECG monitoring on AF management. Search terms were created from the research question.

An initial limited search of PubMed was undertaken to identify articles on the topic. Then, the text words in the titles and abstracts of relevant articles and the index terms used to describe the articles were analysed to identify more relevant search terms. Keywords and index terms used in the search strategy include "atrial fibrillation" AND "mhealth" OR "mobile health" OR "mobile app" OR "mobile application" OR smartphone OR mobile AND "electrocardiography", "electrocardiogram" OR "ECG" OR "monitoring".

A secondary search using all identified keywords and index terms was undertaken on July 26, 2021, across the following databases: PubMed, CINAHL, EMBASE and Google Scholar. In addition, the reference list of all included sources of evidence was screened for additional studies. Studies published since 2010 were included as smartphones with ECG monitoring functions did not exist before 2010.

The two key concepts for this literature review were mobile ECG monitoring and AF management. This review defines mobile ECG as a single-lead ECG obtained by a mobile application using PPG technology or smartphone accessories. Studies were excluded if ECG was not collected through PPG technology or smartphone accessories. For example, studies investigating remote monitoring through an implantable device, such as an implanted cardiac defibrillator, were excluded. In addition, studies focused on AF screening instead of AF detection and management were excluded. Lastly, studies focused on mobile ECG technology validation instead of disease management were also excluded. Due to limited capacity for translation, only studies written in English were considered.

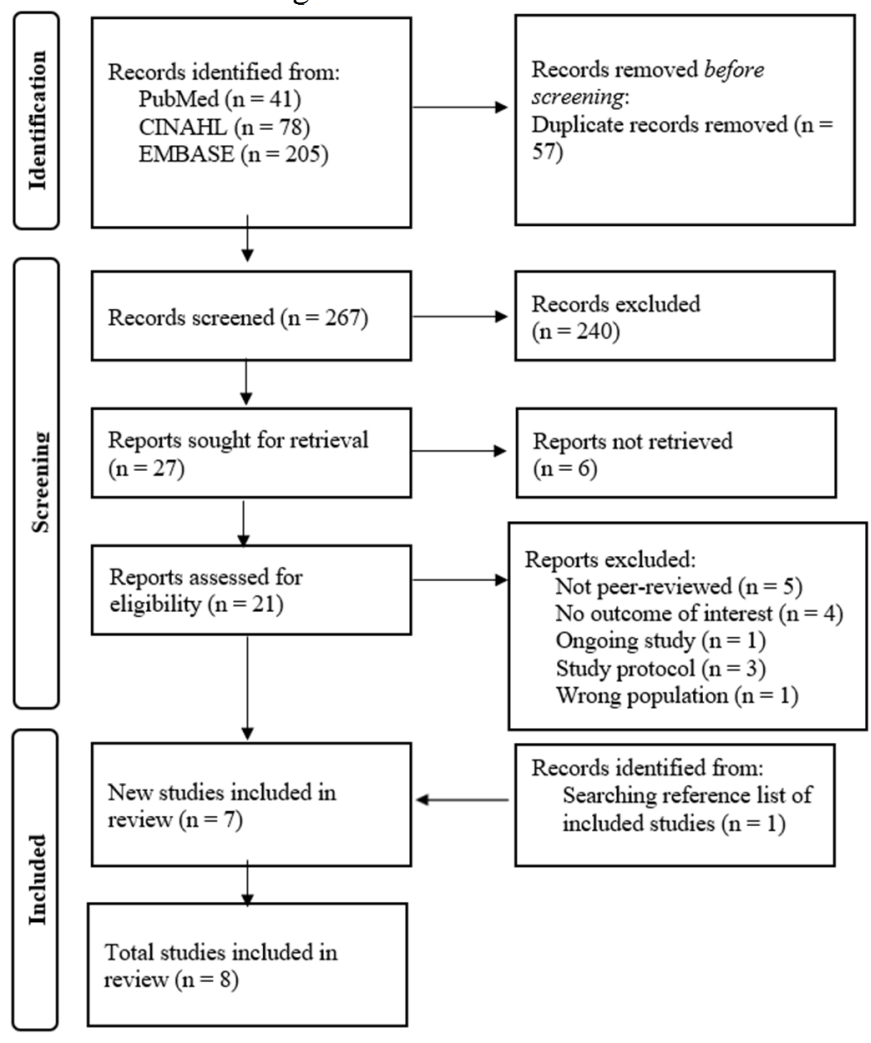

FIGURE 1. Search results and study selection process

The context of this literature review was the globally published peer-reviewed literature on the impact of mobile ECG monitoring on AF management. This review 
considered experimental and quasi-experimental study designs, including randomised controlled trials, nonrandomised controlled trials, before and after studies. In addition, qualitative studies that focused on qualitative data including, but not limited to, designs such as phenomenology and qualitative description were considered.

From the search conducted on July 26, 2021, 324 citations were identified, of which 57 duplicates were removed, leaving 267 records. After screening titles and abstracts, 240 citations were excluded. In addition, full texts of six included articles could not be retrieved. Hence, the full texts of 21 articles were reviewed for further eligibility, of which seven papers met inclusion criteria. One additional study was identified after searching the reference list of all eligible studies. Therefore, the final data set comprised eight studies. The search results are summarised in the PRISMA flow diagram (FIGURE 1). While addressing the proposed objectives of this review, common themes across the included studies were analysed. A summary of common themes is provided in TABLE I.

\section{Results}

\section{A. HEALTH-RELATED QUALITY OF LIFE (HRQOL)}

Two studies from the USA evaluated the effects of mobile ECG monitoring on HRQoL among AF patients [19, 20]. Both studies used the Atrial Fibrillation Effect on Quality of Life (AFEQT) to measure HRQoL. The AFEQT is a validated tool to measure self-reported $\mathrm{HRQOL}$ in $\mathrm{AF}$ patients by assessing 20 different components related to the quality of life in AF patients [27]. Caceres, et al. [19] found that the total AFEQT scores increased in both intervention and control groups. However, intervention group participants had higher scores in all components of AFEQT, while control group participants only demonstrated improvement in symptoms and daily activities [19]. These findings were confirmed in the Guhl, et al. [20] study, which suggested better total AFEQT scores among the intervention group participants. However, although greater improvements in AFEQT were observed in the intervention group, the difference was not statistically significant between intervention and control groups from both studies [19, 20].

\section{B. DETECTING ATRIAL FIBRILLATION RECURRENCE}

Three studies evaluated the effectiveness of mobile ECG monitoring in detecting recurrent AF, of which one study was a randomised control trial [21-23]. The randomised control trial from Goldenthal, et al. [21] demonstrated that mobile ECG monitoring was more effective in detecting recurrent $\mathrm{AF}$ after ablation or cardioversion. AF recurrence was detected in $50.4 \%$ of intervention group participants and $41.5 \%$ of control group participants [21]. Similar results were seen in observational studies from Hermans, et al. [22] and Tarakji, et al. [23], whereby participants were given both Holter and mobile ECG monitoring simultaneously. In Hermans, et al. [22] study, more recurrent AF episodes were identified through mobile ECG monitoring than Holter monitoring (25.2\% vs $14.8 \%$ ). Moreover, Tarakji, et al. [23] demonstrated nearly $100 \%$ sensitivity of mobile ECG monitoring in detecting AF. Furthermore, Goldenthal, et al. [21] found that AF recurrence was identified earlier among participants utilising mobile ECG monitoring. However, both Goldenthal, et al. [21] and Hermans, et al. [22] found that there was no difference in AF recurrence detection rate among intervention and control groups after a certain period ( 1 month in Goldenthal et al.'s vs 14 days in Hermans et al.' s).

\section{REHOSPITALISATION, MORBIDITY AND MORTALITY}

Two studies evaluated the impact of mobile ECG monitoring on hospitalisation $[24,25]$. The randomised controlled trial from Guo, et al. [24] demonstrated that the rehospitalisation rate for participants using mHealth application with ECG monitoring was much lower than participants receiving usual care. The rehospitalisation rates for intervention and control groups were $1.2 \%$ and $4.5 \%$, respectively. Similarly, a retrospective study from Aljuaid, et al. [25] observed a significant reduction in outpatient and emergency

TABLE I.

Themes identified in eligible studies

\begin{tabular}{|c|c|c|}
\hline HRQoL & Caceres, et al. [1]; Guhl, et al. [2] & 2 \\
\hline Detecting AF Recurrence & Goldenthal, et al. [3]; Hermans, et al. [4]; Tarakji, et al. [5]. & 3 \\
\hline $\begin{array}{l}\text { Rehospitalisation, Morbidity \& } \\
\text { Mortality }\end{array}$ & Guo, et al. [6]; Aljuaid, et al. [7]. & 2 \\
\hline Acceptability \& Usability & Guhl, et al. [2]; Hermans, et al. [4]; Tarakji, et al. [5] & 3 \\
\hline $\begin{array}{l}\text { Factors influencing patient } \\
\text { engagement }\end{array}$ & Reading, et al. [8] & 1 \\
\hline
\end{tabular}


department visits among post-ablation patients using mobile ECG monitoring compared with participants using conventional ECG monitoring, such as a Holter monitor. Furthermore, Guo, et al. [24] observed a significant reduction in AF-related co-morbidity such as stroke and heart failure in participants using mHealth application with ECG monitoring compared with the usual care group $(1.9 \%$ vs $6.0 \%$ ). However, although all-cause mortality was lower in the intervention group, the difference was not statistically significant [24].

\section{ACCEPTABILITY \& USABILITY}

Three studies collected patient feedback regarding the acceptability and usability of mobile ECG monitoring through surveys and questionnaires during the final followup [20, 22, 23]. Hermans, et al. [22] reported that more participants gave mobile ECG monitoring a higher rating for usability than Holter monitoring (65.6\% vs $44.3 \%)$. This finding aligned with Tarakji, et al.'s [23] result, which indicated the majority of participants $(92 \%)$ preferred mobile ECG monitoring. Overall, all three studies reported that participants found mobile applications with ECG monitoring functions were easy to use $[20,22,23]$.

\section{E. FACTORS INFLUENCING PATIENT ENGAGEMENT}

A qualitative study from Reading, et al. [26] explored the factors that influence sustained patient engagement in using mobile ECG monitoring. Reading, et al. [26] found that patient characteristics and attitude could influence engagement in using mobile ECG monitoring. Engaged patients demonstrated resilience when self-monitoring AF signs and symptoms. In contrast, unengaged patients became easily discouraged by recurrent AF [26]. When encountering technical issues, engaged patients attempted to troubleshoot. Conversely, unengaged patients often felt frustrated and gave up using mobile ECG monitoring [26]. Furthermore, Reading, et al. [26] reported that the input from healthcare providers was critical for promoting patient engagement. Engaged patients reported adequate feedback and guidance from their health providers, while most unengaged patients reported feeling under-supported [26].

Surprisingly, age was not an identified barrier to using mobile ECG monitoring. On the contrary, Reading, et al. [26] reported that most patients felt confident using the mobile application regardless of age. This finding was confirmed by Hermans, et al. [22], who suggested a strong correlation between older age and increased usage of mobile ECG monitoring.

\section{DISCUSSION}

The mAFA trial was the first randomised control trial to test the effect of a mobile health application on HRQoL in AF patients [28]. The study demonstrated significant improvement in HRQoL in the intervention group. However, the mobile application used in the study did not support ondemand ECG monitoring [28]. Following the mAFA trial, multiple studies have investigated the association between mobile ECG monitoring and HRQoL in AF patients.
Health-related quality of life is a benchmark recognised by international guidelines for AF management [29]. Guhl, et al. [20] evaluated the effect of mobile ECG monitoring on HRQoL among the general AF population, while Caceres, et al. [19] focused on AF patients after catheter ablation and cardioversion. Improved HRQoL among $\mathrm{AF}$ patients is associated with undertaking antiarrhythmic medications or procedures to restore normal sinus rhythm [30, 31]. This explains why both intervention and control groups improved AFEQT in both studies [19, 20].

The randomised control trials from Caceres, et al. [19] and Guhl, et al. [20] did not demonstrate statistical significance in improving AFEQT. Lost to follow-up was reported in both studies, which potentially limited the statistical power of these studies [19, 20]. In addition, these studies only conducted baseline and end-of-study assessments. Increased frequency of assessments throughout a study may improve participant engagement [19]. Further investigation is required in large cohorts of participants and is powered to examine the effects of mobile ECG monitoring on HRQoL in AF after ablation. In addition, strategies need to be developed to reduce missing data in the research methodology. Despite the lack of statistical significance, both Caceres, et al. [19] and Guhl, et al. [20] studies demonstrated more significant improvement of HRQoL in AF patients who utilised mHealth applications with ondemand ECG monitoring functions. Nonetheless, mobile ECG monitoring has the potential to improve HRQoL in AF patients by encouraging timely AF detection and treatment. Barmano, et al. [32] found that recurrent AF is associated with lower HRQoL due to increased AF burden. Mobile ECG monitoring technology facilitates early detection of recurrent $\mathrm{AF}$ and collects valuable ECG data to guide treatment decisions. A recent systematic review from Wong, et al. [33] demonstrated comparable diagnostic accuracy of mobile ECG monitoring compared with traditional Holter monitoring. Both Goldenthal, et al. [21] and Hermans, et al. [22] demonstrated that long-term intermittent mobile ECG monitoring had a better AF recurrence detection rate than short-term continuous Holter monitoring. Interestingly, there was no significant difference in $\mathrm{AF}$ recurrence detection rate using different ECG monitoring methods after 30 days. This finding aligns with the recommendation of up to 30 days of external rhythm monitoring suggested by the Australian Clinical Guidelines for the Diagnosis and Management of Atrial Fibrillation [6]. Thirty days may represent a sufficient monitoring period for AF patients who underwent ablation or cardioversion.

Another potential benefit of implementing long-term intermittent mobile ECG monitoring is engaging patients in AF management [22]. Historically, AF management has focused on the pharmacological interventions provided by medical practitioners. However, there is emerging evidence about the importance of a holistic care approach for providing a patient-centred comprehensive treatment [29]. An integrated AF care model is a holistic AF management approach, broadly defined as a structured healthcare pathway 
that implements guideline-adherent treatment through multidisciplinary input [34]. Hendriks, et al. [35] suggest an integrated care model for AF management should include four components: (1) patient engagement; (2) multidisciplinary approach; (3) use of technology; (4) comprehensive care. Being the centre of integrated AF care, patient engagement is critical. Utilising long-term intermittent mobile ECG monitoring technology engages and empowers patients to monitor their signs and symptoms as part of the AF self-management initiative.

Recent studies have suggested the association between post-ablation AF burden and rehospitalisation [36, 37]. Patients tend to have a higher $\mathrm{AF}$ burden during the "blanking period" after ablation [38]. The "blanking period" refers to the first three months after the ablation, when the heart is healing. During this period, recurrence and associated symptoms are common [38]. Mobile ECG monitoring can potentially reduce AF burden through timely recognition and treatment of recurrent AF. Most mHealth mobile ECG monitoring interventions included in this view allowed participants to receive remote feedback from a healthcare provider regarding their ECG rhythm and symptoms. As a result, the healthcare provider can assess the patient remotely based on the transmitted ECG rhythm and patient symptoms, facilitating early AF recurrence detection and treatment modification. Furthermore, the healthcare provider can also distinguish the symptoms caused by other co-morbidities and provide the patient with relevant healthcare advice to prevent unnecessary emergency department visits [25].

Patient engagement in using mobile ECG monitoring is multifaceted. Factors influencing patient engagement include patient attitude, healthcare provider support, and technology $[39,40]$. The included experimental studies in this review used a range of strategies such as regular text messages and mobile application alerts to maintain patient engagement. Further research is warranted to investigate how to sustain patient engagement in using mobile ECG monitoring for $\mathrm{AF}$ self-management.

Novel mobile ECG monitoring using PPG technology or smartphone accessories offers an alternative rhythm monitoring method with convenience at a low cost [41]. As discussed above, mobile applications with ECG monitoring functions can provide user-friendly on-demand rhythm monitoring, facilitating the early detection of recurrent $\mathrm{AF}$ post-ablation. As a result, incorporating the novel mobile ECG monitoring technology in the guideline-adherent integrated $\mathrm{AF}$ care model may reduce rehospitalisation, improve HRQoL, and better patient clinical outcomes. However, the potential benefit of mobile ECG monitoring is limited to AF detection instead of AF diagnosis. According to the Australian Clinical Guidelines for the Diagnosis and Management of Atrial Fibrillation, a 12-lead ECG is required for accurate diagnosis of AF [6].

Knowledge translation of research evidence into healthcare practice is complex. It can be influenced by multiple factors, including the quality of evidence, the context of the healthcare system, and the views and beliefs of healthcare professionals [42]. This review indicates emerging evidence demonstrating the benefits of utilising mobile ECG monitoring for post-ablation AF management. In addition, most Australians are smartphone owners and can become potential users of mobile health applications. Furthermore, the government initiative of integrating electronic healthcare records into the healthcare system worldwide has led to the increasing use of digital health interventions for chronic disease management [11]. However, this novel model of rhythm monitoring is relatively foreign for many healthcare professionals. Therefore, adequate education and facilitation regarding mobile ECG monitoring need to be provided to healthcare professionals who will implement this intervention in clinical practice. This literature review would serve as an excellent educational resource to facilitate the knowledge translation of this novel rhythm monitoring technology for AF management.

This literature review has limitations. Firstly, studies included in this review had predominant white participants, which means the findings from this review may not apply to a culturally diverse population. Secondly, only studies published in English were included. It is possible that some relevant studies were missed.

\section{CONCLUSION}

This review provided an overview of emerging evidence about the impact of mobile ECG monitoring on AF management. The review analysed and synthesised the emerging evidence about the utilisation of mobile ECG monitoring for AF management. Key concepts that underpin the research field of mobile ECG monitoring for AF management were identified. Mobile ECG monitoring seems to positively impact $\mathrm{HRQ}$ oL, hospital readmission rates, morbidity, and mortality in AF patients. Novel mobile ECG monitoring using PPG technology or smartphone accessories offers an alternative rhythm monitoring method with convenience at a low cost. Further research is warranted to investigate how best to sustain patient engagement in using mobile ECG monitoring for AF self-management.

\section{REFERENCES}

[1] N. R. Jones, C. J. Taylor, F. R. Hobbs, L. Bowman, and B. Casadei, "Screening for atrial fibrillation: a call for evidence," European Heart Journal, vol. 41, no. 10, pp. 1075-1085, 2020, doi: 10.1093/eurheartj/ehz834.

[2] J. Kornej, E. J. Benjamin, and J. W. Magnani, "Atrial fibrillation: global burdens and global opportunities," Heart, vol. 107, no. 7, pp. 516-518, 2021, doi: 10.1136/heartjnl-2020-318480.

[3] G. Lippi, F. Sanchis-Gomar, and G. Cervellin, "Global epidemiology of atrial fibrillation: An increasing epidemic and public health challenge," International Journal of Stroke, vol. 16, no. 2, pp. 217 221, 2021, doi: 10.1177/1747493019897870.

[4] Australian Institute of Health and Welfare. "Atrial fibrillation in Australia." AIHW. https://www.aihw.gov.au/reports/heart-strokevascular-diseases/atrial-fibrillation-in-australia (accessed 16 August 2020).

[5] R. B. Schnabel et al., "50 year trends in atrial fibrillation prevalence, incidence, risk factors, and mortality in the Framingham Heart Study: 
a cohort study," The Lancet, vol. 386, no. 9989, pp. 154-162, 2015, doi: 10.1016/S0140-6736(14)61774-8.

[6] D. Brieger et al., "National Heart Foundation of Australia and Cardiac Society of Australia and New Zealand: Australian clinical guidelines for the diagnosis and management of atrial fibrillation 2018," Medical Journal of Australia, vol. 209, no. 8, pp. 356-362, 2018, doi: 10.1016/j.hlc.2018.06.1043.

[7] A. Verma et al., "Approaches to catheter ablation for persistent atrial fibrillation," New England Journal of Medicine, vol. 372, no. 19, pp. 1812-1822, 2015, doi: 10.1056/NEJMoa1408288.

[8] N. Varma et al., "HRS/EHRA/APHRS/LAHRS/ACC/AHA worldwide practice update for telehealth and arrhythmia monitoring during and after a pandemic: Developed in partnership with and endorsed by the American College of Cardiology (ACC), the American Heart Association (AHA), the Asia Pacific Heart Rhythm Society (APHRS), the European Heart Rhythm Association (EHRA), the Heart Rhythm Society (HRS), and the Latin American Heart Rhythm Society (LAHRS)," EP Europace, vol. 23, no. 2, pp. 313313, 2021, doi: 10.1093/europace/euaa187.

[9] M. P. Turakhia and D. W. Kaiser, "Transforming the care of atrial fibrillation with mobile health," Journal of Interventional Cardiac Electrophysiology, vol. 47, no. 1, pp. 45-50, 2016, doi: 10.1007/s10840-016-0136-3.

[10] P. Guzik and M. Malik, "ECG by mobile technologies," Journal of Electrocardiology, vol. 49, no. 6, pp. 894-901, 2016, doi: 10.1016/j.jelectrocard.2016.07.030

[11] D. Kotecha et al., "European Society of Cardiology smartphone and tablet applications for patients with atrial fibrillation and their health care providers," EP Europace, vol. 20, no. 2, pp. 225-233, Feb 1 2018, doi: 10.1093/europace/eux299.

[12] Australian Bureau of Statistics. "Household use of information technology."

https://www.abs.gov.au/statistics/industry/technology-andinnovation/household-use-information-technology/latest-release (accessed 2021).

[13] World Health Organization. "mHealth. New horizons for health through mobile technologies." WHO. https://www.who.int/goe/publications/goe_mhealth_web.pdf (accessed.

[14] A. Walker and J. Muhlestein, "Smartphone electrocardiogram monitoring: current perspectives," Advanced Health Care Technologies, vol. 4, pp. 15-24, 2018, doi: 10.2147/ahct.S138445.

[15] C. R. Lopez Perales et al., "Mobile health applications for the detection of atrial fibrillation: a systematic review," EP Europace, vol. 23, no. 1, pp. 11-28, 2021, doi: doi:10.1093/europace/euaa139.

[16] K. H. C. Li et al., "The current state of mobile phone apps for monitoring heart rate, heart rate variability, and atrial fibrillation: narrative review," JMIR mHealth and uHealth, vol. 7, no. 2, p. e11606, 2019, doi: 10.2196/11606.

[17] Y. Guo et al., "Mobile health technology-supported atrial fibrillation screening and integrated care: A report from the mAFA-II trial Longterm Extension Cohort," European Journal of Internal Medicine, vol. 82, pp. 105-111, Dec 2020, doi: 10.1016/j.ejim.2020.09.024.

[18] D. A. Lane et al., "Mobile health applications for managing atrial fibrillation for healthcare professionals and patients: a systematic review," EP Europace, vol. 22, no. 10, pp. 1567-1578, 2020, doi: 10.1093/europace/euaa269.

[19] B. A. Caceres et al., "Mobile Electrocardiogram Monitoring and Health-Related Quality of Life in Patients With Atrial Fibrillation: Findings From the iPhone Helping Evaluate Atrial Fibrillation Rhythm Through Technology (iHEART) Study," Journal of Cardiovascular Nursing, vol. 35, no. 4, pp. 327-336, Jul/Aug 2020, doi: 10.1097/JCN.0000000000000646.

[20] E. Guhl et al., "The Atrial Fibrillation Health Literacy Information Technology Trial: Pilot Trial of a Mobile Health App for Atrial Fibrillation," JMIR Cardio, vol. 4, no. 1, p. e17162, Sep 4 2020, doi: $10.2196 / 17162$.

[21] I. L. Goldenthal et al., "Recurrent atrial fibrillation/flutter detection after ablation or cardioversion using the AliveCor KardiaMobile device: iHEART results," Journal of Cardiovascular Electrophysiology, vol. 30, no. 11, pp. 2220-2228, Nov 2019, doi: 10.1111 /jce.14160.
[22] A. N. Hermans et al., "Long-term intermittent versus short continuous heart rhythm monitoring for the detection of atrial fibrillation recurrences after catheter ablation," International Journal of Cardiology, vol. 329, pp. 105-112, 2021, doi: 10.1016/j.ijcard.2020.12.077.

[23] K. G. Tarakji et al., "Using a novel wireless system for monitoring patients after the atrial fibrillation ablation procedure: the iTransmit study," Heart Rhythm, vol. 12, no. 3, pp. 554-559, 2015, doi: 10.1016/j.hrthm.2014.11.015.

[24] Y. Guo et al., "Mobile Health Technology to Improve Care for Patients With Atrial Fibrillation," Journal of American College of Cardiology, vol. 75, no. 13, pp. 1523-1534, Apr 7 2020, doi: 10.1016/j.jacc.2020.01.052.

[25] M. Aljuaid et al., "Smartphone ECG monitoring system helps lower emergency room and clinic visits in post-atrial fibrillation ablation patients," Clinical Medicine Insights: Cardiology, vol. 14, p. 1179546820901508, 2020, doi: 10.1177/1179546820901508.

[26] M. Reading, D. Baik, M. Beauchemin, K. T. Hickey, and J. A. Merrill, "Factors Influencing Sustained Engagement with ECG SelfMonitoring: Perspectives from Patients and Health Care Providers," Applied Clinical Informatics, vol. 9, no. 4, pp. 772-781, Oct 2018, doi: $10.1055 / \mathrm{s}-0038-1672138$.

[27] J. Spertus et al., "Development and validation of the Atrial Fibrillation Effect on QualiTy-of-Life (AFEQT) Questionnaire in patients with atrial fibrillation," Circulation: Arrhythmia and Electrophysiology, vol. 4, no. 1, pp. 15-25, 2011, doi 10.1161/CIRCEP.110.958033.

[28] Y. Guo, Y. Chen, D. A. Lane, L. Liu, Y. Wang, and G. Y. H. Lip, "Mobile Health Technology for Atrial Fibrillation Management Integrating Decision Support, Education, and Patient Involvement: mAF App Trial," The American Journal of Medicine, vol. 130, no. 12, pp. 1388-1396 e6, Dec 2017, doi 10.1016/j.amjmed.2017.07.003.

[29] P. Kirchhof et al., "2016 ESC Guidelines for the management of atrial fibrillation developed in collaboration with EACTS," Kardiologia Polska (Polish Heart Journal), vol. 74, no. 12, pp. 1359-1469, 2016 , doi: 10.1016/j.recesp.2016.11.014.

[30] D. B. Mark et al., "Effect of catheter ablation vs medical therapy on quality of life among patients with atrial fibrillation: the CABANA randomized clinical trial," JAMA, vol. 321 , no. 13, pp. 1275-1285, 2019, doi: 10.1001/jama.2019.0692.

[31] N. J. Sethi, J. Feinberg, E. E. Nielsen, S. Safi, C. Gluud, and J. C. Jakobsen, "The effects of rhythm control strategies versus rate control strategies for atrial fibrillation and atrial flutter: A systematic review with meta-analysis and Trial Sequential Analysis," PLOS ONE, vol. 12, no. 10, p. e0186856, 2017, doi: 10.1371/journal.pone.0186856.

[32] N. Barmano, E. Charitakis, J. E. Karlsson, F. H. Nystrom, H Walfridsson, and U. Walfridsson, "Predictors of improvement in arrhythmia - specific symptoms and health - related quality of life after catheter ablation of atrial fibrillation," Clinical Cardiology, vol. 42, no. 2, pp. 247-255, 2019, doi: 10.1002/clc. 23134

[33] K. C. Wong, H. Klimis, N. Lowres, A. von Huben, S. Marschner, and C. K. Chow, "Diagnostic accuracy of handheld electrocardiogram devices in detecting atrial fibrillation in adults in community versus hospital settings: a systematic review and meta-analysis," Heart, vol. 106, no. 16, pp. 1211-1217, 2020, doi: 10.1136/heartjnl-2020316611.

[34] D. A. Lane and G. Y. Lip, "Integrated care for the management of atrial fibrillation: what are the key components and importan outcomes?," Ep Europace, vol. 21, no. 12, pp. 1759-1761, 2019, doi: 10.1093/europace/euz211.

[35] J. M. Hendriks, H. J. Crijns, and H. J. Vrijhoef, "Integrated chronic care management for patients with atrial fibrillation-a rationale for redesigning atrial fibrillation care," Journal of Atrial Fibrillation, vol. 7, no. 5, 2015, doi: 10.4022/jafib.1177.

[36] C. Alhede et al., "Higher burden of supraventricular ectopic complexes early after catheter ablation for atrial fibrillation is associated with increased risk of recurrent atrial fibrillation," $E p$ Europace, vol. 20, no. 1, pp. 50-57, 2018, doi 10.1093/europace/euw329.

[37] J. J. Liang et al., "Early recurrence of atrial arrhythmias following pulmonary vein antral isolation: Timing and frequency of early 
recurrences predicts long-term ablation success," Heart Rhythm, vol. 12, no. 12, pp. 2461-2468, 2015, doi: 10.1016/j.hrthm.2015.07.015.

[38] S. Willems et al., "Redefining the blanking period after catheter ablation for paroxysmal atrial fibrillation: insights from the ADVICE (Adenosine Following Pulmonary Vein Isolation to Target Dormant Conduction Elimination) trial," Circulation: Arrhythmia and Electrophysiology, vol. 9, no. 8, p. e003909, 2016, doi: 10.1161/CIRCEP.115.003909.

[39] Y. K. Dwivedi, N. P. Rana, A. Jeyaraj, M. Clement, and M. D. Williams, "Re-examining the unified theory of acceptance and use of technology (UTAUT): Towards a revised theoretical model," Information Systems Frontiers, vol. 21, no. 3, pp. 719-734, 2019, doi: 10.1007/s10796-017-9774-y.
[40] V. Venkatesh, J. Y. Thong, and X. Xu, "Unified theory of acceptance and use of technology: A synthesis and the road ahead," Journal of the Association for Information Systems, vol. 17, no. 5, pp. 328-376, 2016.

[41] M. R. Turchioe, V. Jimenez, S. Isaac, M. Alshalabi, D. Slotwiner, and R. M. Creber, "Review of mobile applications for the detection and management of atrial fibrillation," Heart Rhythm O2, vol. 1, no. 1, pp. 35-43, 2020, doi: 10.1016/j.hroo.2020.02.005.

[42] G. Harvey and A. Kitson, "PARIHS revisited: from heuristic to integrated framework for the successful implementation of knowledge into practice," (in eng), Implement Science, vol. 11, p. 33, Mar 10 2016, doi: 10.1186/s13012-016-0398-2. 